\title{
Duplicações pieloureterais e ureteroceles da criança- terapêutica cirúrgica
}

\author{
Ureteral duplications and ureteroceles - surgical treatment
}

\author{
Lisieux Eyer de Jesus ${ }^{1}$, Mariana Mesquita Júdice², Eduardo G. Mello²
}

\section{Resumo}

Objetivo: estudar as manifestações clínicas e radiológicas e o tratamento cirúrgico das duplicações pieloureterais sintomáticas na criança, especialmente com relação ao resultado das heminefrectomias polares superiores.

Material e métodos: estudo prospectivo de observação num grupo de 15 pacientes pediátricos, tratados cirurgicamente, segundo um protocolo, com seguimento mínimo de 6 meses pós-operatórios.

Resultados: $93 \%$ dos pacientes se apresentaram, no primeiro ano de vida, por infecção urinária e/ou hidronefrose fetal. Heminefrectomia polar superior foi a cirurgia mais freqüente (80\%). Todos os pacientes obtiveram melhora sintomática e radiológica após o procedimento, que não apresentou complicações significativas. As três crianças portadoras de refluxo vesicoureteral grave para o segmento superior da duplicação apresentaram refluxo sintomático para o coto ureteral no pós-operatório e sofreram ureterectomia distal. Nenhuma das crianças sem refluxo vesicoureteral pré-operatório necessitou de um segundo tempo cirúrgico.

Conclusões: a heminefrectomia polar superior é um método seguro e eficaz para o tratamento das duplicações pieloureterais sintomáticas da criança. Em pacientes que não apresentam refluxo vesicoureteral associado, provavelmente será a única cirurgia necessária. No caso de pacientes portadores de refluxo vesicoureteral severo para o pólo superior de duplicação, poderá ser necessária ureterectomia distal posteriormente.

J Pediatr (Rio J) 2003;79(2):173-80: duplicação urinária, heminefrectomia, refluxo vesicoureteral, coto ureteral, ureterocele.

\section{Introdução}

O tratamento das duplicações sintomáticas do trato urinário da criança é de alta complexidade e persiste muito controverso. Várias propostas terapêuticas são recentes, em especial as que usam a incisão endoscópica sistemática

1. TCBC/RJ, Cirurgiã Pediátrica do Hospital Municipal Jesus, RJ e do Hospital Universitário Antônio Pedro, UFF, RJ.

2. R2 do Serviço de Cirurgia Pediátrica do Hospital Municipal Jesus.

Artigo submetido em 27.11.02, aceito em 31.01.03

\begin{abstract}
Objective: to study clinical and radiological data and surgical treatment for symptomatic ureteral duplications in childhood, especially the results for superior polar heminephrectomies.
\end{abstract}

Methods: prospective observational study concerning 15 pediatric patients surgically treated following a protocol with a minimum follow-up of 6 months.

Results: $93 \%$ of the patients presented urinary infection and/or fetal hydronephrosis during their first year of life. These were the usual reasons for evaluation. Superior polar heminephrectomy was indicated for $80 \%$ of the patients, all of whom presented clinical and radiological improvement after the surgery. There were no significant complications. All children with severe vesicoureteral reflux to the duplicated superior pole presented symptomatic reflux to the stump after the surgery and were submitted to stumpectomy. None of the patients without preoperative reflux needed any other surgical procedure.

Conclusions: superior polar heminephrectomy is safe and efficient in order to treat ureteral duplications in childhood. If the patient does not present vesicoureteral reflux this will probably be the only surgery needed. Most patients with reflux to the resected superior pole will need stumpectomy subsequently.

J Pediatr (Rio J) 2003;79(2):173-80: ureteral duplication, heminephrectomy, vesicoureteral reflux, ureteral stump, ureteroceles.

das ureteroceles como terapia inicial ${ }^{1,2}$, com avaliação ainda incompleta dos resultados em longo prazo. Esta metodologia, muito atraente do ponto de vista de sua simplicidade num primeiro momento, em alguns casos causará refluxo vesicoureteral severo, de tratamento difícil.

A maioria dos estudos disponíveis em literatura é retrospectivo. Não encontramos estudos publicados com casuísticas brasileiras, e muitos trabalhos limitam períodos muito longos de tempo, durante os quais os algoritmos de conduta 
clínica sofreram alterações, determinando dificuldades na uniformização dos resultados. Apresentamos uma casuística em pacientes pediátricos ( $<12$ anos) estudada de forma prospectiva, destacando aspectos clínicos e de diagnóstico, em nosso meio, e os resultados, particularmente no que concerne às heminefrectomias polares superiores. Nosso principal objetivo foi o de registrar a expressão clínica e os aspectos diagnósticos das duplicações urinárias sintomáticas em nosso meio, e verificar a capacidade resolutiva e eventuais complicações das heminefrectomias polares superiores como tratamento para as duplicações pieloureterais. O protocolo escolhido determina o tratamento do trato urinário como um todo, sem individualizar o tratamento das ureteroceles, consideradas, por nós, parte integrante de um complexo de malformações anatômicas, cujo conjunto, em detrimento de cada componente individual, determinará as conseqüências clínicas e seu tratamento.

\section{Casuística e métodos}

Apresentamos um estudo caso controle prospectivo, realizado num período de 5 anos (1996-2001), em 15 crianças, operadas pelo mesmo cirurgião, no serviço de cirurgia pediátrica do Hospital Municipal Jesus, Rio de Janeiro, Brasil, com período mínimo de observação pósoperatória de 6 meses. Trata-se de hospital pediátrico de nível terciário, funcionando como referência para cirurgia pediátrica complexa na Secretaria de Saúde do município do Rio de Janeiro.

Como critério para inclusão nesta casuística, foi adotado o diagnóstico de duplicação ureteral completa sintomática. As crianças foram avaliadas inicialmente através de ultra-sonografia (US), uretrocistografia miccional (UCM), cintilografia renal (CGR) e urografia excretora (PIV). As indicações cirúrgicas foram infecção urinária (ITU) de repetição ou intratável e/ou suboclusão da saída vesical ou dos ureteres.

Duplicações com ausência de função significativa do segmento polar superior foram submetidas à heminefrectomia do pólo superior, por lombotomia, com ressecção do ureter correspondente, na máxima extensão possível, sem nova incisão e laqueadura da extremidade proximal do segmento residual de ureter (12 casos). Doentes com boa função polar superior e sem refluxo vesicoureteral demonstrado (1 caso) sofreram bypass ureteropiélico términolateral superior-inferior, com ressecção máxima do coto ureteral distal do pólo superior e anastomose do coto ureteral proximal à pelve renal inferior. Pacientes com boa função polar superior e refluxo vesicoureteral sintomático não resolvido através de antibioticoprofilaxia profilática e observação prolongada ( $\geq 2$ anos), ou com problemas associados que exigissem tratamento cirúrgico, receberam reimplante ureteral duplo, após ressecção de ureterocele (um caso). Casos com função renal ipsilateral desprezível foram nefrectomizados (um caso). Todas as cirurgias foram realizadas a céu aberto, e nenhuma criança recebeu tratamento endoscópico pré-operatório.
Todos os pacientes foram agendados para seguimento conforme um protocolo prevendo quimioprofilaxia antibiótica, preferencialmente usando sulfametoxazol associado ao trimetropin $(40 \mathrm{mg} / \mathrm{kg}$ sulfametoxazol $+8 \mathrm{mg} / \mathrm{kg}$ trimetropin em dose única noturna), em maiores de 4 meses de idade, e cefalexina $25 \mathrm{mg} / \mathrm{kg}$, dose única noturna, nos lactentes de primeiro trimestre, durante os três primeiros meses pós-operatórios e estendida, se necessário, segundo critérios clínicos (diagnóstico de refluxo vesicoureteral intercorrente, ocorrência de ITU na ausência do quimioterápico). Todos foram agendados para revisão ambulatorial clínica e ultra-sonográfica sistemática, independentemente da presença de sintomas, ou de sinais de anormalidades, com 1, 3, 6 meses e 1 ano pós-operatório e vigilância clínica ambulatorial por um mínimo de 5 anos. Os responsáveis foram instruídos a contactar o serviço em caso de infecções febris e/ou sintomas urológicos intercorrentes. CGR e UCM eram solicitadas após um mínimo de quatro meses pósoperatórios.

Todos os responsáveis foram informados quanto à necessidade eventual de um segundo tempo cirúrgico, para tratamento de seqüelas da doença (em geral refluxo vesicoureteral - RVU), quando as crianças foram submetidas à cirurgia inicial sobre os segmentos renais superiores.

\section{Resultados}

Foram estudadas 15 crianças entre 3 e 50 meses de idade (mediana 7 e média 13,5 m), 11 meninas e 4 meninos (relação 2,8:1), com seguimento pós-operatório mínimo de 6 meses (variância 6 a 77 meses, média 24,7 meses, mediana 16 meses). Cinco apresentavam doença à direita, e 10, à esquerda; dois apresentavam duplicações ureterais bilaterais com manifestações patológicas apenas em um dos rins duplicados.

Quatro pacientes (27\%) tiveram diagnóstico pré-natal de anomalia urológica, mas não receberam quimioprofilaxia antibiótica ou investigação radiológica após o nascimento, sendo encaminhadas ao serviço após episódio de pielonefrite aguda, à exceção de um, inicialmente assintomático, encaminhado para investigação de hidronefrose fetal, que apresentou sintomas (ITU) posteriormente, apesar do uso de quimioprofilaxia. Oito enfermos (53\%) apresentaram sintomas até os 3 meses de idade, três até os 6 meses e um até 1 ano de vida, num total de $73 \%$ de casos sintomáticos no primeiro semestre, e $93 \%$, no primeiro ano de vida. Apenas uma criança apresentou os primeiros sintomas em idade pré-escolar. A média para a expressão clínica das anomalias foi de 8,3 meses e mediana de 3 meses. Apesar do quadro clínico precoce, houve uma latência significativa entre os sintomas e o primeiro atendimento especializado, determinada em 13 doentes, variando entre menos de 3 meses, a partir da sintomatologia inicial (7/13), e mais de 1 ano, após o primeiro episódio de infecção (2/13). 4/13 crianças foram atendidas apenas entre 6 meses e 1 ano, após as primeiras manifestações clínicas. O período 
entre a primeira avaliação médica adequada e o tratamento cirúrgico foi maior que 6 meses em $9 / 13$ pacientes, com média de 13,8 meses e mediana de 21 .

Em 14/15 crianças, o motivo do encaminhamento foi um ou mais episódios de ITU febril ou ITU persistente/ "intratável". A única exceção foi um neonato assintomático encaminhado para investigação de hidronefrose fetal. O primeiro episódio de ITU foi verificado durante o primeiro mês de vida em dois casos, no primeiro trimestre em 6 e até o sexto mês em 9. Os demais apresentaram o primeiro episódio no primeiro ano de vida (3 casos), e apenas 2 meninas (13\%) apresentaram sintomas infecciosos iniciais acima de 1 ano. Em um doente foi impossível determinar o momento do primeiro episódio de infecção. As médias e medianas de idade observadas para o primeiro episódio de ITU foram, respectivamente, de 10,4 e 6 meses, 13,7 e 5 meses e 4,8 e 6 meses, em geral, no sexo feminino e no masculino. O encaminhamento das meninas para investigação foi mais tardio que para os meninos (média de 15,2 e mediana 11 meses para meninas e 7 meses para meninos). Onze dos 15 pacientes observados haviam apresentado mais de 2 episódios de ITU febril, quando encaminhados (variância de 3 a 8 episódios reconhecidos), e 1 apresentouse após 2 meses de tratamento, mediante vários esquemas terapêuticos, com ITU persistente "intratável". Em apenas 3 , houve $\leq 2$ episódios de ITU severa, prévios ao diagnóstico.

Dois pacientes apresentaram retenção urinária, ambos do sexo masculino e portadores de ureterocele, um deles neonato com prolapso de ureterocele para uretra posterior, referido como caso de ITU intratável. Duas meninas apresentavam corrimento vaginal. Não houve nenhum caso de prolapso de ureterocele em menina, incontinência urinária, orquiepididimite ou ectopia ureteral perineal.

Todas as crianças foram investigadas com US, UCM e PIV. CGR foi realizada em $87 \%$ ( $n=13)$. Foi difícil uniformizar os dados de exames, uma vez que não houve padronização metodológica para execução e descrição dos mesmos. No caso de pacientes submetidos a mais de um exame do mesmo tipo no pré-operatório, foram integradas as informações.

O exame de US cita explicitamente duplicação em apenas $5 / 15$ enfermos, embora a presença desta possa ser deduzida indiretamente, ou pressuposta em 8 dos demais (descrição de 2 ureteres de um mesmo lado, ureterocele ectópica, megaureter "superior"), e o diagnóstico de duplicação pôde ser afirmado ou suposto, apenas pela US, em $87 \%$ dos casos. Em quatro pacientes foi necessário mais de um exame para detectar a doença, e a US não foi capaz de diagnosticar o problema em dois, perfazendo um total de 6/15 em que um exame de US inicial não foi satisfatório para detectar duplicação. A US descreveu lesão do parênquima renal em seis crianças, 12 hidronefroses no pólo superior (1 pionefrose) e seis hidronefroses do pólo inferior de duplicação, nenhum caso de hidronefrose contralateral. Este exame demonstrou-se altamente sensível para detectar megaureter associado, descrevendo-o em todos os doentes, mas relacionando a anomalia ao pólo superior de duplicação em apenas sete. Em 11/15 casos, a US detectou ureteroceles, inclusive três não detectadas pela UCM, e descreveu 3/15 ectopias ureterais (comparada a 4 detectadas pela $\mathrm{UCM})$.

CGR com DMSA foi feita em 13 pacientes, demonstrando diminuição da massa renal ipsilateral, ou exclusão do pólo superior do rim ipsilateral em 77\% ( $\mathrm{n}=10)$. Em 3 (23\%) doentes, havia função do pólo superior ipsilateral, embora uniformemente diminuída, e em apenas um, disfunção significativa do pólo inferior. Uma criança (8\%) mostrou cicatriz renal em pólo inferior ipsilateral. O rim contralateral era normal em 12/13 e aumentado em 1/13 (duplicação bilateral). As CGR com DTPA ( $n=13)$ mostraram exclusão do pólo superior e hidronefrose do pólo inferior de duplicação em nove casos, com hidronefrose contralateral em dois. A função do rim doente era, em $56 \%$ dos examinados, significativamente menor que a do homólogo (diferença de função entre os dois lados > 10\%).

A UCM demonstrou sensibilidade menor que a US para detectar ureteroceles (Tabela 1). 4/15 pacientes, dois portadores de ureterocele ao US, tiveram UCM normal. Em 11 crianças sabidamente portadoras de ureteroceles, a US identificou 10 (sensibilidade de 91\%), e a UCM, 7 (sensibilidade de 64\%). Apenas um bebê tinha bexiga de esforço (sexo masculino, obstrução urinária por prolapso de ureterocele), mas houve aumento do resíduo pós-miccional em $3 / 15(20 \%)$ enfermos, todos portadores de ureteroceles. Nenhum foi descrito no US, porém não foi feito exame vesical pós-miccional sistemático. A UCM é insubstituível para detecção de refluxo vesicoureteral (RVU) ipsilateral em 5 (33\%) pacientes (4 para pólo superior e 3 para pólo inferior, $3 / 4$ refluxos grau 5 para o pólo superior), e contralateral em 2 (13\%) (grau 3), demonstrando-se uma incidência anormalmente elevada de RVU nesta população específica (incidência média em lactentes de 1 a $2 \%{ }^{3}$ ). A UCM foi capaz de desmonstrar a presença de ectopia ureteral (em ureteres refluentes correspondentes ao pólo superior de duplicação) em 4 pacientes. Todos estes pacientes apresentavam os ureteres ectópicos muito dilatados e inseridos próximos ao colo vesical.

A PIV demonstrou pólo superior excluso em 11/15 exames (em 7 indiretamente, através do "sinal da flor murcha"). Dois pacientes com pólo superior excluso na PIV tiveram função demonstrada na cintigrafia. Um paciente apresentou exclusão renal ipsilateral total (nefrectomizado). Foi detectada obstrução funcional pólo inferior ipsilateral em apenas $4 / 15$ pacientes, contra $9 / 15$ na cintigrafia com DTPA. A PIV foi capaz de detectar 5 casos de ureteroceles, todos previamente vistos através da UCM e US.

Em 12 doentes, heminefrectomia polar superior, ureterectomia máxima e ligadura da extremidade residual do ureter foi a abordagem escolhida. Houve um bypass ureteropiélico súpero-inferior, um reimplante ureteral duplo a 
Tabela 1 - Comparação entre a capacidade de detecção de problemas específicos em exames complementares

\begin{tabular}{lllll}
\hline Parâmetro & US (\%) & UCM (\%) & PIV (\%) & Cintigrafia (\%) \\
\hline Anomalia do ureter terminal & $13 / 15(87)$ & $8 / 15(53)$ & 0 & - \\
Ureterocele & $11 / 15(73)$ & $8 / 15(53)$ & $5 / 15(33)$ & - \\
Ectopia ureteral & $3 / 15(20)$ & $4 / 15(27)$ & 0 & - \\
Megaureter & $15 / 15(100)$ & $5 / 15(33)$ & 0 & 0 \\
Duplicação pieloureteral & $13 / 15(87)$ & Indireto & $11 / 15(73)$ & $4 / 13(31)$ \\
Hidronefrose pólo superior ipsilateral & $11 / 15(73)$ & - & $5 / 15(3)$ & $9 / 13(69)$ \\
Hidronefrose pólo inferior ipsilateral & $6 / 15(40)$ & - & $10 / 15(67)$ & $9 / 13(69)$ \\
Exclusão pólo superior ipsilateral & - & - & $5 / 15(33)$ & $4 / 13(31)$ \\
Função pólo superior ipsilateral & - & - & 0 & $2 / 13(15)$ \\
Hidronefrose contralateral & 0 & - & &
\end{tabular}

Politano e uma nefrectomia total. Houve problemas pósoperatórios em 3 crianças: 1 pneumonia, 1 ITU (pionefrose do pólo superior de duplicação por Pseudomonas aeruginosa detectada no ato cirúrgico), 1 cistite asséptica autolimitada (reimplante ureteral, ressecção ureterocele e reconstrução do assoalho vesical). $\mathrm{O}$ tempo de internação variou entre 3 e 9 dias (média: 6,1; mediana: 6).

Em 12 casos, houve peças anatômicas para revisão histológica. Onze apresentavam pielonefrite crônica e alterações inflamatórias e obstrutivas. Apenas 3 pólos superiores $(25 \%)$ foram descritos como displásicos, 1 como portador de glomeruloesclerose, e 3, como atróficos (25\%).

O seguimento variou entre 6 e 77 meses (média 24,7 e mediana 16 meses). Em vários doentes com medidas seriadas, há um processo de crescimento renal proporcionalmente maior no lado operado com relação ao contralateral. Em 14/15 crianças não foi descrita anormalidade no segmento renal remanescente nas ultra-sonografias pós-operatórias (9/15 apresentavam padrão obstrutivo no pólo inferior de duplicação no pré-operatório, que presumimos ter sido devido à compressão secundária da(s) desembocadura(s) ureteral(is) pela ureterocele). $O$ paciente restante demonstrou melhora progressiva da hidronefrose verificada no pré-operatório.

Dos enfermos submetidos à heminefrectomia, 2/13 apresentaram cistos de pequeno volume (diâmetro máximo $<20$ $\mathrm{mm}$ ), assintomáticos, sobre o segmento renal remanescente. Nenhum dos 11 portadores de ureterocele mostra a lesão em pós-operatório, porém são visíveis espessamentos vesicais localizados em quatro, e um desenvolveu (72m após nefrectomia total) um divertículo vesical no sítio da ureterocele anteriormente descrita, com refluxo no coto ureteral.

UCM pós-operatória está disponível em 10/15 doentes. É normal em 3 (assintomáticos), mostra refluxo de coto em
3 (apresentaram ITU pós-operatória), refluxo para pólo inferior ipsilateral em 3 (2 com ITU pós-operatória e 1 assintomático) e refluxo contralateral em 1 (assintomático).

Seis pacientes estão clinicamente assintomáticos entre 6 e 60 meses pós-operatórios. Nove outros apresentaram ITU (entre 2 e 72 meses, mediana 10 meses). Houve justificativas anatômicas para todos 7 casos: $3 / 9$ apresentavam RVU para coto ureteral residual (ITU com 8, 12 e 72 meses de pós-operatório), 1/9 hidrocolpos (perda de seguimento), 3/9 RVU para pólo inferior ipsilateral e/ou contralateral (1 perda de seguimento), 2/9 aguardam investigação complementar.

Três casos de refluxo severo para o pólo superior de duplicação apresentaram a síndrome do coto residual de ureter, com ITU e episódios de disúria pós-operatória e foram reoperados (ureterectomia distal extravesical), persistindo, desde então, assintomáticos. Entre os que não apresentavam RVU pré-operatório, há três casos de RVU "novo" (um assintomático e dois casos de ITU pós-operatória). Quatro crianças não foram submetidas ainda à UCM pós-operatória, e três persistem sem RVU.

5/15 (33\%) pacientes persistem assintomáticos no pósoperatório, nenhum dos quais exibiu em qualquer momento evidências de RVU. Mais dois casos apresentaram refluxo "novo" no pós-operatório, também resolvido espontaneamente após período de observação e quimioprofilaxia, perfazendo um total de 7/15 (47\%) curados após uma única intervenção. Dois doentes persistem em observação, um deles sem diagnóstico pré-operatório de RVU, com um único episódio pós-operatório de ITU, aguardando UCM. Quatro doentes (27\%) foram reoperados, três para ressecção de coto residual de ureter (permanência hospitalar 24h) e um para cura de RVU (cirurgia por solicitação de familiares, que não aceitam quimioprofilaxia em longo prazo). 
Apenas dois enfermos (13\%) perderam o seguimento, ambos meninas sintomáticas: um caso de RVU "novo" pósoperatório, e um caso sem RVU pré e pós-operatório, com US diagnosticando hidrocolpos. Um resumo dos dados de seguimento pode ser observado na Tabela 2 .

\section{Discussão}

Consideramos a casuística apresentada representativa, apesar do pequeno número de casos, frente ao pequeno número de estudos prospectivos disponíveis ${ }^{1-2,5} \mathrm{e}$ à ausência de estudos nacionais quanto ao tratamento das duplicações complicadas do trato urinário na criança, após extensa pesquisa usando a base de dados BIREME. O tema é controverso, e algumas formas de tratamento foram apresentadas muito recentemente, não há ainda seguimento em longo prazo para algumas modalidades, notadamente endoscopia terapêutica. As principais controvérsias envolvem o dilema entre endoscopia terapêutica versus a capacidade de cura definitiva, e métodos de maior risco operatório (cirurgias de reconstrução vesical extensa) versus métodos de menor morbidade cirúrgica (heminefrectomia), mas uma menor eficácia para cura definitiva em tempo único. Cabe aqui ressaltar que a endoscopia diagnóstica tem indicações já clássicas, e que a controvérsia com relação à endoscopia terapêutica não se aplica para casos de obstrução da saída vesical ou infecção perinatal irresponsiva à antibioticoterapia ótima, na qual o tratamento endoscópico inicial, sempre que disponível, não é discutido.

Em nosso meio, persistem dificuldades de diagnóstico e encaminhamento precoce dos pacientes. Quando houve diagnóstico intrauterino de anomalia urinária, este, na mai- oria das vezes, não foi acompanhado de encaminhamento para investigação e tratamento, em quatro pacientes (27\%) diagnosticados através de US fetal, apenas um foi encaminhado precocemente e assintomático. Um segundo problema diz respeito ao diagnóstico específico tardio, após o início da sintomatologia, com uma média de 13,5 meses (mediana 7 meses) entre o início dos sintomas e o encaminhamento para investigação urológica, e pela ocorrência de mais de um episódio de ITU previamente à avaliação especializada e instituição de quimioprofilaxia. Este problema foi mais grave para o sexo feminino (mediana de idade da primeira avaliação para meninas de 11 meses, e de 7 meses para meninos). É possível que esta diferença possa ser atribuída à conduta antiga, atualmente reformulada ${ }^{3,6-7}$, que preconizava investigação no primeiro episódio de ITU no sexo masculino e apenas após dois episódios para meninas. Também é possível que haja um viés para diagnóstico mais precoce em meninos em face de quadro clínico de maior gravidade ou controle mais difícil. O encaminhamento e investigação tardia representam um ônus com relação a seqüelas de ITU de repetição e sepse. É necessário conscientizar os profissionais atuantes na área de obstetrícia, pediatria e cuidados primários à saúde quanto à necessidade de investigação precoce em crianças suspeitas de malformação urológica, ou que apresentem ITU, imediatamente após o primeiro episódio. Também é necessário melhorar os cuidados pré-natais, em face do pequeno número de pacientes com diagnóstico intra-útero (27\%), embora os dados em literatura internacional sejam freqüentemente semelhantes $\left(28 \%{ }^{8}, 17 \%^{9}, 31 \%^{10}, 25 \%^{5}, 52 \%^{4}, 58 \%^{11}\right)$, considerando as evidências de que o diagnóstico pré-natal permite uma diminuição significativa na incidência de ITU.

Tabela 2 - Resumo do seguimento clínico pós-operatório

\begin{tabular}{lllll}
\hline Paciente & FU* $(\mathbf{m})$ & ITU pós-operatória & Quadro clínico associado & Tratamento \\
\hline 1 & 4 & Não houve & Assintomático & Desnecessário, paciente assintomático \\
2 & 16 & $3 \mathrm{~m}$ & Sinéquia vulvar/ RVU, PID e CL & Reimplante ureteral bilateral \\
3 & 14 & $3 \mathrm{~m}$ & Refluxo coto & Cirurgia 11m pós-operatório \\
4 & 13 & $12 \mathrm{~m}$ & Aguardando UCM & Quimioprofilaxia \\
5 & 12 & $8 \mathrm{~m}$ & Hidrocolpos & Perda seguimento \\
6 & 7 & $2 \mathrm{~m}$ & RVU(4), PID & Perda seguimento \\
7 & 28 & Não houve & Assintomático, RVU(2), IL3(CL) & Quimioprofilaxia, cura 15m \\
8 & 18 & Não houve & Assintomático & Desnecessário, paciente assintomático \\
9 & 40 & $8 \mathrm{~m}$ & Refluxo coto & Cirurgia 12m \\
10 & 37 & $8 \mathrm{~m}$ & Assintomático, RVU(2), IL & Observação clínica \\
11 & 6 & Não houve & Assintomático & Desnecessário, paciente assintomático \\
12 & 35 & Não houve & Assintomático & Desnecessário, paciente assintomático \\
13 & 4 & $2 \mathrm{~m}$ & Assintomático & Quimioprofilaxia \\
14 & 60 & Não houve & Assintomático & Desnecessário, paciente assintomático \\
15 & 77 & $72 \mathrm{~m}$ & Refluxo coto & Cirurgia 74m pós-operatório \\
\hline
\end{tabular}

(*) FU: Follow up, tempo de seguimento. PID: pólo inferior de duplicação, IL: ipsilateral, CL: contralateral. 
A uniformização inadequada dos dados de exames complementares nos causou grande dificuldade, e teria sido ideal protocolar a execução e o registro destes exames. Ainda assim, é possível concluir que a US é extremamente eficaz pelo baixo custo, morbidade mínima e alta sensibilidade. Nesta série, a US foi o método de imagem mais eficaz para o diagnóstico de ureteroceles, inclusive com relação às UCMs, embora estas sejam indispensáveis para a detecção de RVU (presente em até $50 \%$ dos pacientes ${ }^{12}$ ). Provavelmente a melhor positividade do US frente à UCM se explica pela compressibilidade da maior parte das ureteroceles, que podem não se evidenciar na bexiga sob repleção máxima. Embora nesta casuística tenham havido problemas com o diagnóstico direto de duplicação, supostamente isto ocorreu devido à ausência de padronização da execução e descrição do exame. O estudo pré e pós-miccional, do qual não dispusemos, seria interessante para a detecção de fenômenos suboclusivos da saída vesical, secundários a ureteroceles ectópicas. Uma nova vertente de utilização da US é a verificação indireta do potencial funcional de pólos superiores de duplicação em neonatos, nos quais as provas funcionais atualmente disponíveis são de baixa eficácia. A avaliação quanto à espessura e características sonográficas do parênquima renal pela US pode ser o melhor recurso disponível.

A PIV não acrescentou dados às informações obtidas através dos demais exames complementares (US/ UCM/ cintigrafia), e obriga algumas conclusões a serem feitas de forma indireta. No caso de duplicação com pólo superior excluso, habitual em pediatria, a PIV corrobora o diagnóstico através de sinais radiológicos dependentes de fenômenos mecânicos de deslocamento renal (sinal da flor murcha, deslocamento lateral do ureter do pólo inferior), através da visualização de um sistema pielocaliceal incompleto no pólo inferior de duplicação, ou demonstrando o sistema polar superior através de refluxo vesicoureteral espontâneo (o que pode causar uma falsa impressão de funcionalidade no pólo superior, fato que suspeitamos explicar algumas discordâncias verificadas entre DMSA, DTPA e PIV). Em nossa opinião, a avaliação funcional obtida pela PIV pode ser suprida com vantagens pela cintigrafia renal e a informação anatômica através de exame ultra-sonográfico de boa qualidade técnica, relegando este exame a um plano secundário. Reconhecemos, no entanto, que, em face da ausência de padronização do exame de US e/ou de operador qualificado, ou ainda em presença de dúvida diagnóstica, a PIV persiste como recurso valioso.

Observamos uma incidência relativamente pequena de displasia renal no segmento polar superior de duplicação (25\%), embora a maioria dos autores relate índices em torno de metade dos $\operatorname{casos}^{8,13-15}$. Este dado sofre a ausência de padronização na definição de displasia renal pelos patologistas ${ }^{16}$. Isso pode ser problemático, especialmente por que alguns argumentam - em defesa de uma abordagem resseccional do pólo superior excluso - que a extirpação destes segmentos seria necessária em face das possíveis complicações potencialmente presentes em segmentos displásicos. Se a frequiência de displasia for pequena, associando-se a informações comprovando incidência de neoplasia ou hipertensão, semelhantes à população geral em portadores de displasia renal, ${ }^{17-19}$ tal argumentação deverá ser desconsiderada.

Verificamos melhora clínica em todos os pacientes operados. A morbidade cirúrgica foi baixa, tivemos apenas um caso de problema ligado estritamente à técnica cirúrgica (cistite e espasmos vesicais de repetição em reimplante ureteral). Em pacientes submetidos à heminefrectomia, as complicações foram ligadas à entubação orotraqueal (pneumonia pós- operatória precoce) e à infecção crônica do sistema urinário.

Não há evidência de prejuízo funcional ou déficit de crescimento renal em nenhum pólo inferior remanescente, embora tal possibilidade seja uma argumentação teórica empregada por vários autores. Embora tenhamos verificado tamanho menor no rim remanescente, do lado operado, com relação ao contralateral, o remanescente renal apresenta em todos os pacientes tamanho compatível com o padrão normal para faixa etária em nomograma, sempre que esteve disponível mensuração ( 9 pacientes). Estes dados estão de acordo a literatura, que apresenta função significativa do pólo superior de duplicação apenas em cerca de 1/4 dos casos, e com Vates ${ }^{15}$, que, em análise funcional de pacientes com duplicação renal, não verificou índices de função global significativamente diferentes entre casos de ressecção e casos de preservação polar superior (diferenças de função renal global no pós-operatório de $+2,3$ a $\pm 2,3 \%$ em heminefrectomias e $-1,3$ a $\pm 2,7 \%$ em pacientes que tiveram o pólo superior preservado, com relação aos valores pré-operatórios). Anormalidades funcionais, na forma de suboclusão funcional do segmento renal inferior ou do rim contralateral, se normalizaram (14/15 casos), ou apresentaram significativa melhora (1/15 caso). É possível que o tratamento endoscópico perinatal apresente resultados melhores quanto a preservar/restaurar a função renal nos segmentos polares superiores, com bons resultados em $1 / 5^{1,8}$ a 2/3 dos casos ${ }^{2,20-21}$, porém tais dados ainda dependem de tempo para se provar efetivos, e será necessário comprovar a importância relativa da função polar superior preservada no total da função renal disponível para o paciente, inclusive frente a eventuais problemas secundários ao uso da endoscopia (em especial indução de RVU severo).

Verificamos pequenos cistos sobre o segmento renal remanescente, em região correspondente à linha de sutura renal, em dois pacientes, fato que não encontramos relatado em literatura. Tais lesões persistem assintomáticas, e talvez seja possível sua resolução espontânea, possivelmente constituindo cistos de retenção. Todas as ureteroceles se resolveram espontaneamente sem seqüelas, exceto em um paciente, que passou a apresentar divertículo vesical periureteral após um prazo muito longo de observação (6 anos). 
Aproximadamente metade dos pacientes estão assintomáticos no pós-operatório, dois com RVU de baixo grau. Em nenhum, havia RVU demonstrado no pré-operatório. Estes dados estão de acordo com Hussman 22 , que apresenta heminefrectomia como cirurgia única em $85 \%$ dos pacientes sem RVU pré-operatório, com $30 \%$ de RVU novo pósoperatório e $15 \%$ de reimplantes, contra $84 \%$ de RVU persistente em portadores de RVU pré-operatório e $57 \%$ de RVU e reimplantes secundários, após endoscopia terapêutica perinatal. Em 6/7 pacientes que apresentaram problemas pós-operatórios foi possível detectar uma causa, dois casos de RVU para o pólo inferior de duplicação ou rim contralateral, três casos de refluxo para coto ureteral residual (um deles associado a divertículo periureteral), um caso de malformação genital associada (um paciente aguarda UCM). Em todos os pacientes sintomáticos no pós-operatório, com uma única exceção, a sintomatologia residual se apresentou no primeiro ano de seguimento, rapidamente, após a suspensão da quimioprofilaxia antibiótica. Nossa incidência de refluxo para coto residual de ureter foi semelhante à da literatura ${ }^{10-11,23-25}$, e todos os casos foram reoperados com incisão à Pfannestiel e ressecção extravesical do coto ureteral residual, com permanência hospitalar pós-operatória e seqüela estética mínimas, inclusive supondo que, havendo disponibilidade de cirurgia laparoscópica, tal abordagem se torne ainda mais simples.

Em nossa opinião, a possibilidade de complicações obriga ao seguimento longo, e é necessária pelo menos uma UCM pós-operatória, embora alguns autores recomendem o exame apenas em caso de sintomatologia clínica.

Verificamos problemas pós-operatórios em todos os casos em que foi reconhecido RVU pré-operatório. A necessidade de reoperação tem sido colocada como o grande fator contrário à utilização da abordagem "mínima" através de heminefrectomia proposta por Cendron ${ }^{26}$, e modernamente a endoscopia tem sido apresentada como nova terapia de intervenção mínima. No entanto, o tratamento endoscópico é reconhecidamente indutor de RVU (35 a 90\% dos casos) $)^{3,4,8,9,20,22}$, que necessitará de tratamento cirúrgico complementar, desviando o eixo da atenção cirúrgica da heminefrectomia para a cirurgia de reimplante e exigindo atenção redobrada quanto à profilaxia antibiótica enquanto aguarda cirurgia, o que pode ser difícil em condições sociais adversas.

Concluímos que o tratamento, por heminefrectomia, das duplicações complicadas, com exclusão funcional do pólo superior, é um bom recurso terapêutico, e a única intervenção necessária em pacientes que não apresentem RVU. No caso de pacientes apresentando refluxo para o pólo superior duplicado, possivelmente será necessária a ressecção do segmento ureteral residual, embora este segundo tempo consista em cirurgia de pequena monta e, no intervalo entre as intervenções, se verifique grande melhora clínica e anatômica dos segmentos renais remanescentes. Para pacientes com refluxo pré-operatório contralateral ou para segmento inferior de duplicação, as chances de reope- ração são intermediárias, e estarão na dependência de problemas anatômicos na implantação vesical dos ureteres remanescentes e/ou distúrbios funcionais da bexiga, e na capacidade de seguimento do paciente sob quimioprofilaxia em longo prazo.

\section{Referências bibliográficas}

1. Petit T, Ravasse P, Delmas P. Does the endoscopic incision of ureteroceles reduce the indications for partial nephrectomy? BJU International 1999;83:675-8.

2. Jayanthi VR, Koff AS. Long-term outcome of transurethral puncture of ectopic ureteroceles: initial success and late problems. J Urol 1999;162:1077-80.

3. Rushton HG. Urinary tract infections in children. Epidemiology, evaluation and management. Pediatr Clin North Am 1997;44(5):1133-69.

4. Konus OL, Ozdemir A, Akkaya A, Erbas G, Celik H, Isik S. Normal liver, spleen and kidney dimensions in neonates, infants and children: evaluation with sonography. Am J Roentgenol 1998;171:1693-8.

5. Kropp BP, Cheng EY, Parrott T, Kropp K. Primary lower tract reconstruction for non-functioning renal moieities. Soc Pediatric Urology newsletter 2001;2(1). Disponível em: URL: http:// www.spu.org.

6. Larcombe J. Clinical evidence: urinary tract infection in children. BMJ 1999;319:1173-5.

7. Ferrer FA, McKenna PH, Hochman HI, et al. Results of a vesicoureteral reflux practice pattern survey among American Academy of Pediatrics, section on pediatric urology members. J Urol 1998;160:1031-7.

8. Monfort G, Guys JM, Coquet M, Roth K, Louis C, Bocciardi A. Surgical management of duplex ureteroceles. J Pediatr Surg 1992;27(5):634-8.

9. Choi H, Oh SS. The management of children with ureteral complete duplication: selective use of ureterostomy as a primary and salvage procedure. BJU International 2000;86:508-12.

10. Plaire JC, Pope JC 4th, Kropp BP, Adams MC, Keating MA, Rink RC, et al. Management of ectopic ureters: experience with the upper tract approach. J Urol 1997;158:1245-7.

11. Ade-Ajayi N, Wilcox DT, Duffy PG, Ransley PG. Upper pole heminephrectomy: is complete ureterectomy necessary? BJU International 2002;88:77-9.

12. Caldamone AA, Sbyder HM, Duckett JW. Ureteroceles in children: follow up and management with upper tract approach. J Urol 1987;131:1130-4.

13. Sen S, Beasley SW, Ahmed S, Smith ED. Renal function and vesicoureteral reflux in children with ureteroceles. Pediatric Surg Intern 1992;7:192-4.

14. Smith FL, Ritchie EL, Maizels M, Zaontz MR, Hsueh W, Kaplan WE, et al. Surgery for duplex kidneys with ectopic ureters: ipsilateral ureteroureterostomy versus polar nephrectomy. J Urol 1989;142:532-4.

15. Vates TS, Bukowski T, Triest J, Freedman A, Smith C, Perlmutter $\mathrm{A}$, et al. Is there a best alternative to treating the obstructed upper pole? J Urol 1996;156:744-6.

16. Pantuck, AJ, Fleischer, MH. Pyelonephritis complicating a multicystic dysplastic kidney. Infect Urol 2000;13(1):16-7.

17. Perez LM, Naidu SI, Joseph DB. Outcome and analysis of operative versus nonoperative management of neonatal multicystic dysplastic kidneys. J Urol 1998;160:1207-11. 
18. Rootenberg GT, Gordon I, de Bruyn R. The natural history of the multicystic dysplastic kidney in children. Br J Radiol 1997; 70:347-50.

19. Suktankar S, Watson AR. Unilateral multicystic displastic kidney disease: defining the natural history. Acta Pediatr 2000;89:811-3.

20. Singh SJ, Smith G. Effectiveness of primary endoscopic incision of ureteroceles. Pediatr Surg Intern 2001;17(7):528-31.

21. Chertin B, Fridmans A, Hadas-Halpren I, Farkas A. Endoscopic puncture of ureterocele as a minimally invasive and effective long-term procedure in children. Eur Urol 2001;39(3):332-6.

22. Husmann D, Strand B, Ewalt D, Clement M, Kramer S, Allen T. Management of ectopic ureterocele associated with renal duplication: a comparison, of partial nephrectomy and endoscopic decompression. J Urol 1999;162:1406-9.

23. Culp OS. Hemineprhoureterectomy: comparison of one-stage and two-stage operations. J Urol 1960;83:369-71.

24. Barrett DM, Malek RS, Kelalis PP. Problems and solutions in surgical treatment of 100 consecutive ureteral duplications in children. J Urol 1975;114:126-30.
25. Zubieta RA, Castilho RA, Encalada R, Escala J, Ramirez R, Field M. Experiencia de diez años: ureterocele abandonado. Ver Chil Urol 1996;61(1):152-3.

26. Cendron J, Melin Y, Valayer J. Traitement simplifié de l'urétérocèle aved duplicité piélo-urétérale chez l'enfant. A propos de 35 cas. Chir Pédiatr 1980;21:121-4.

Endereço para correspondência:

Dr. Lisieux Eyer de Jesus

Rua Presidente Domiciano, 52/ 801 - Boa Viagem

CEP 21210-270 - Niterói, RJ

Fone: (21) 2622.3843

E-maillisieux@uol.com.br 